\title{
Spin First vs. Position First instructional approaches to teaching introductory quantum mechanics
}

\author{
Homeyra R. Sadaghiani \\ California State Polytechnic University, Department of Physics and Astronomy, 3801 W. Temple Ave., Pomona, CA, 91768
}

\begin{abstract}
As part of ongoing research in teaching and learning quantum mechanics, we are investigating student learning of basic introductory quantum concepts in two different paradigms. In one paradigm, students are introduced to the postulates of quantum mechanics by discrete bases of Spin-half (Spin First) before being introduced to Schrödinger's equation. In the second paradigm, continuous bases of position probability wave functions (Position First) are the context within which students first encounter quantum mechanical phenomena. In this paper, we compare student learning of basic introductory quantum ideas in two sections of a sophomore level modern physics course at $\mathrm{Cal}$ Poly Pomona that were taught using these two approaches by means of their performances on a research-based concept posttest. Based on our results, the students who were taught using Spin First outperformed their peers in Position First group with average score of $53 \pm 3 \%$ vs. $34 \pm 5 \%$ in Quantum Mechanics Concept Assessment (QMCA), suggesting that the Spin First approach might improve some aspects of student learning of quantum mechanics.
\end{abstract}

\section{INTRODUCTION}

Learning quantum mechanics $(\mathrm{QM})$ is challenging for many students in sophomore level courses. Aside from its unconvincing philosophical interpretations for some students, the extremely abstract concepts along with complex mathematical formalisms in this course are often beyond the level of sophistication most students encounter prior to their first QM courses [1]. Furthermore, the thorough mental engagement in successful sense making and problem-solving processes in these courses demand students to simultaneously draw upon multiple conceptual and mathematical resources. This process can be overwhelming for naïve learners and could increase their cognitive load to the extent in which they are unable to process or retain any new information [2].

We have been investigating how different QM curricular approaches might impact student learning of various quantum mechanical topics using two different paradigms: Spin First (SF) vs. Position First (PF). The SF approach uses the sequential Stern-Gerlach Experiments (SGE) [9] with discrete bases of spin-half as a context to introduce the postulates of quantum mechanics, whereas the PF approach starts by utilizing the Schrödinger equation and the continuous basis of position probability wave functions to solve problems related to the energy and position of a particle in various potential wells. Although over the past decade, substantial research and curriculum development has taken place on QM both at the introductory and advanced undergraduate levels [3], previous research on the effectiveness of the Spin First approach is extremely limited. Nevertheless, the research that does exist on use of SGE as a tool has shown promising results. For example, such an approach has shown to increase students abilities to differentiate between physical and Hilbert spaces [4] and recognize superposition states from mixtures [5]. Other research suggests using computer simulations of SGE as an effective way to push students towards grappling with the concepts behind the postulates of $\mathrm{QM}$, rather than simply teaching them to mechanically solve problems [6].

We have been studying various implementations of SF ap- proach in teaching QM courses at Cal Poly Pomona since Fall 2011. Our previous results [7] suggest that use of the Spin First approach does not hinder students' learning and it may in fact improve their understanding of certain topics. For example, the SF approach made a positive impact on modern physics students' overall average scores on the subset of Quantum Mechanics Concept Assessment (QMCA) [8] as compared to those in the PF group, and although the differences reported in other data compared (e.g., midterm and final exams, and Quantum Mechanics Concept Survey) were not significant, they all showed a positive trend in favor of the SF approach.

In this paper, we present additional data with an enhanced controlled environment where both sections of the course were taught by the same instructor, resulting in better controlled variables and consistency between the two classes. Student learning of basic topics of introductory quantum ideas with either the SF or PF approaches is compared by means of their performances on a common midterm examination as a pretest and a subset of the QMCA questions as a posttests. We present students' over all performance as well as their average scores in individual questions on the QMCA in more detail.

\section{SPIN FIRST \& POSITION FIRST PARADIGMS}

Through the analysis of sequential Stern-Gerlach spin experiments, using two state spin-half systems, the SF curriculum examines the postulates of QM prior to any discussion of Schrödinger's equation [9]. In this approach, Dirac and bra-ket notation for states, observables, and projection operators are used from the very beginning. Students are asked to use matrix representations of operators and given opportunities to make explicit connections between the Dirac and matrix representations while analyzing concrete experimental results. After in-depth discussions of key quantum mechanical principles in the context of spin-half, the SF approach utilizes these principles in the discussion and calculation of the wave function of a particle in various potential wells. This 
TABLE I. The curriculum and schedule comparison for Spin First (SF) and Position First (PF) at Cal Poly Pomona.

\begin{tabular}{lll}
\hline \hline Week & Position First & Spin First \\
\hline 1 & Birth of Modern Physics, Blackbody Radiation & Birth of Modern Physics, Blackbody Radiation \\
2 & Plank Quantization, Photoelectric Effect & Plank Quantization, Photoelectric Effect \\
3 & Structure of Atom, Thomson \& Rutherford Models & Structure of Atom, Thomson \& Rutherford Models \\
4 & Bohr Model, X-Ray, Spectra & Bohr Model, X-Ray Spectra \\
5 & De-Broglie Waves, Wave Particle Duality & De-Broglie Waves, Wave Particle Duality \\
6 & Double Slits \& Electron Scattering & Polarization, Spin Magnetic Moment \\
7 & Wave Function, Properties, Normalization & Dirac Notation, Postulate, Stern-Gerlach Experiments \\
8 & Schrödinger Equation, Probability, Expectation Values & Matrix Notation, Quantum State Vectors \\
9 & Infinite \& Finite Potential Well & Schrödinger Equation, Particle in a Box, Expectation Values \\
10 & Barriers \& Quantum Tunneling & Finite Potential Well, Barriers \& Quantum Tunneling \\
\hline \hline
\end{tabular}

approach allows students to first focus on abstract concepts where the mathematics are simple and limited to calculations related to two-by-two matrices.

In the PF approach, QM concepts are introduced in the context of the wave function of a particle in various onedimensional-potential wells with continuous bases of position probability densities. The postulates of quantum mechanics are not usually taught explicitly in this approach [10] and focus often tends to be on carrying out repeated mathematical calculations rather than aiming for sense making based on experimental results. This approach requires students to simultaneously engage with abstract concepts and mathematics; however, it often emphasizes skills for solving complex integrals and differential equations, with little or no explicit discussion of underlying quantum mechanical principles.

\section{BACKGROUND}

Cal Poly Pomona's PHY 235 course, which is a required course for physics majors and minors at the sophomore level, is designed to introduce students to the origins of quantum physics; Bohr theory; atomic structure; wave mechanics, and the introduction to quantum mechanics. The course used to be offered only once in Spring quarter; however, due to an increase in student demand in the last two years, the course is being offered twice and sometimes three times a year. PHY 235 generally meets 150 minutes a week in a lecture format (three 50 minute or two 75 minute periods), along with a separate weekly three-hour laboratory session.

The previous study, in which the data was collected from several sections of modern physics and upper level undergraduate QM classes that were taught in the SF or PF approach at Cal Poly Pomona, showed a small but positive impact on students' performances in the classes taught using the SF approach [7]. However, due to logistics and implementation issues, the variables for the two instructional paradigms were not optimally controlled. For example, the course instructors were not the same and thus used different textbooks, course materials, and various degrees of interactive engagement strategies in class. In the present data set, we have been able to reduce the number of uncontrolled variables between the two sections.

\section{RESEARCH STUDY}

The present study focuses on data from two sections of PHY 235 courses in the 2015-16 academic year that were both taught by the author using the two different PF or SF paradigms. The two courses followed the same textbook [12] for most topics, particularly during the discussion of the experimental bases of QM (weeks 1 - 5) and before the midterm examinations. During the next three weeks (weeks 6 - 8) students in the SF group diverged from the course textbook and were introduced to the postulates of quantum mechanics using spin-half systems and computer simulations of sequential SGE. They also began using bra-ket and Dirac notation and matrix mechanics. Meanwhile, a greater time was allocated to the discussion of the probability density, properties of wave function, and its graphical representations in the PF class. During this time, various class examples of common integrals for normalization and expectation value calculations were also discussed in detail in the PF class. The topics discussed for the rest of the quarter (weeks 9 - 10) were very similar but offered at slightly different paces and depth as briefly outlined in TABLE I. We are aware that alternative mix, order, and focus in topics could have been designed and that this may not be an ideal curriculum nor schedule.

\section{A. Methodology}

Many aspects of the two courses including similar interactive pedagogical strategies (peer instruction, group work, discussion format, etc.) and course material for the common topics (homework, quiz, class examples, concept tests, and problem solving examples) were identical or slightly altered 


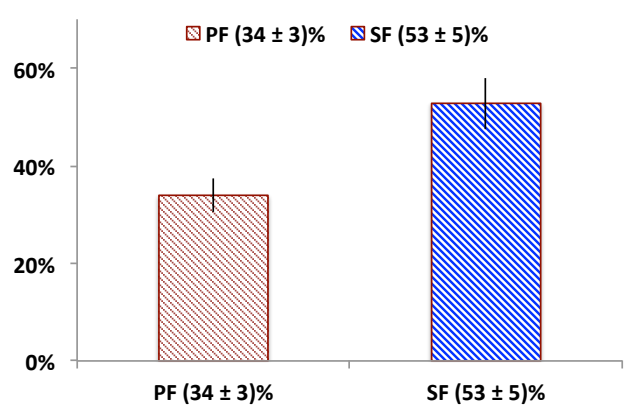

FIG. 1. 14-item QMCA posttest scores for the Spin First (SF) and Position First (PF). The error bars represent the standard errors.

while presented in different orders, pace, and focus. The Fall 2015 course with 22 students utilized SF approach while the Spring 2016 course with 44 students was taught by PF approach.

The Quantum Mechanics Concept Assessment (QMCA) [8] is a 31-item conceptually-focused survey used as a posttest to evaluate students' knowledge of five main topics in quantum mechanics, including, quantum measurement (Meas), the time-independent Schrödinger equation (TISE), wave functions and boundary conditions (WF), time evolution (TE), and probability density (Prob). We have previously identified a 14-item subset of QMCA questions [7] to be appropriate for use in sophomore level quantum courses. The five QMCA concept categories on the 14-item QMCA subset used in this study included Meas. (7 questions), TISE (5 questions), WF (8 questions), TE (2 questions), and Prob. (4 questions). Some questions fell into more than one conceptual category. Almost all the QMCA questions are written in the context of spatial wave function of particles in onedimensional potential wells and use no Dirac notation. At the end of the quarter, we administered the QMCA subset in class to students in both courses to measure the relative effectiveness of the two different instructional methods. The questions in this subset include (Q1-Q7) \& (Q22-Q28) from the 31-item survey [13] . An identical midterm exam served as a pretest where no significant differences were found in student average scores (SF: $69 \pm 5 \%$; PF: $66 \pm 3 \%$ ).

\section{B. Results}

Only 18 out of the 22 students in Fall 2015 and 36 out of the 44 students in Spring 2016 completed the QMCA posttest. The overall average score of the students in the SF class on the subset of QMCA questions was (53 \pm 3$) \%$, while the average score for the PF was $(34 \pm 5) \%$. Figure 1 shows that students in the SF group outperformed the PF group by $19 \%$ ( 0.9 Effect size). An independent-sample t-test suggests a significant difference in the overall average score for the two groups (P $<0.05)$. These results are consistent with previously reported data [7] when the controlled and treatment courses had more

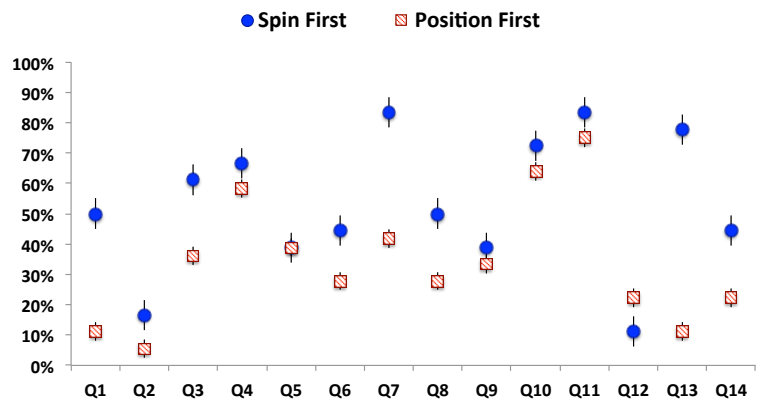

FIG. 2. Student average scores on individual questions for the 14item QMCA in the Spin First (SF) and Position First (PF). The average score for Q5 was identical for the two groups.

unmatched variables (SF: $N=19,54 \pm 5 \%$; PF: $N=22$, $37 \pm 4 \%, 0.6$ Effect size).

The analysis of student scores on the fourteen individual questions in Fig. 2 shows that the SF group outscored their peers in the PF group in all questions except Q5 and Q12. The average score of the students in the two paradigms were identical for Q5 (39)\% while PF group outscored SF group by $11 \%$. Q12 on the 14-item subset corresponds to question number twenty-six on the 31-item QMCA and asks students to approximate the probability of finding a particle confined to $0<x<a$, in a state given by $\psi(x)=\frac{1}{\sqrt{2}}\left[\psi_{1}(x)+\psi_{2}(x)\right]$ in the first half of the region, using the graphical representation of the two normalized wave functions $\psi_{1}(x)$ and $\psi_{2}(x)$. This topic and similar examples were discussed in more depth in PF course than the SF class. On the other hand, SF students scored much higher on questions $1,3,7,13$, and 14 (27 \& 28 on 31-item QMCA). TABLE II presents the percentage difference in the average scores of the students in SP and PF group as well as the related concept frame work of these questions. While the detailed discussion of these individual questions is outside the scope of this proceeding and will be presented in future publications, it is important to note that four out of the five questions fall under measurement concept framework of QMCA.

A second posttest measurement was performed during the last week of classes using the 12-item Quantum Mechanics Concept Survey[16]. No significant difference was found on students overall average scores in SF and PF classes (SF: $(55 \pm 3) \%, N=19$ vs. PF: $(57 \pm 3) \%, N=44)$. This is not surprising because the QMCS questions are mainly focused on the topics discussed in the earlier part of this course and prior to the midterm examination (weeks $1-5$ ), indicating that the use of the SF approach in the second part of the course did not affect students performances on the questions related to earlier topics. 
TABLE II. The top five questions and related topics in which the Spin First group outscored their peers in the Position First group.

\begin{tabular}{clc}
\hline \hline Q & Concept Framework & (SF-PF) \% \\
\hline 1 & Meas. \& Prob. & $39 \%$ \\
3 & Meas. & $25 \%$ \\
7 & TISE. \& WF & $42 \%$ \\
13 & Meas. & $67 \%$ \\
14 & Meas. \& WF & $22 \%$ \\
\hline \hline
\end{tabular}

\section{DISCUSSION}

Some motivations and justifications for the potential effectiveness of a SF approach include the following: concrete and robust experimental foundation, the inherently quantum mechanical nature of spin of a particle, and limited (perhaps simpler) mathematical skill requirements related to discrete bases. First, the two-state spin-half system utilized in this approach is one of the simplest, yet most instructive examples with experimental bases. The concrete experimental results from the sequential SGE apparatus are utilized to introduce students to the most mind bending concepts ranging from quantum measurement and probability, to superposition and time-evolution of quantum states. Secondly, the pure quantum mechanical nature of spin could potentially aid students' effective constructions of the new framework needed for analyzing the microscopic phenomena. Nonclassical nature of spin could reduce the likelihood of activating student resources based on their prior experience with the macroscopic world, some of which are proposed to destructively interfere with the acquisitions of QM ideas [11]. Thirdly, the SF approach involves only two discrete bases of spinhalf and deals with a limited number of measurement outcomes mostly using math skills related to two-by-two matrix multiplications. Whereas the PF approach involves continuous bases of probability wave functions with many possible measurement outcomes, all of which demand more advanced mathematics, such as solutions to second order differential equations and various integrals: skills many of our sophomore undergraduates have not mastered yet.

Other variables include the explicit discussion of the QM postulates in SF or the repeated mechanical problem solv- ing recipes common to calculations in PF, where students with strong mathematical background could cruise through the course successfully with avoiding the sense making process. Many students who are capable of computing integrals, often lack conceptual understanding of their underlying meaning [14].

Cognitive load theory seems to be an appropriate framework that could be applied to this study. According to Sweller, conventional problems impose a heavy cognitive load which does not assist in learning [2]. In fact, literature indicates that the cognitive load imposed on a person solving complex problems can negatively interfere with their learning by preventing them from noticing essential aspects of a problem's underlying concepts and structure [15]. Therefore, considering that the simultaneous demand for use of complex math with abstract concepts (and ability to connect the two) places a large cognitive load on students when learning $\mathrm{QM}$, devoting their entire cognitive processing capacity to the mathematical aspect of problems can exclude those features necessary for sense making.

Although a direct measure of the cognitive load imposed by either of these teaching approaches is not available currently, one would expect any potential measure would simultaneously account for number of variables, need for any prior knowledge, problem difficulty, and strategy used. The larger number of variables involved in the continuous bases of the $\mathrm{PF}$ approach as well as required advanced mathematics could increase the problem difficulty levels, resulting in a greater cognitive load. Thus, the heavy use of limited cognitiveprocessing capacity during more advanced mathematical calculations involved in learning QM through the PF approach may leave no room for schema acquisition and interpretations of the problem solutions. Meanwhile, a mathematically less demanding approach (e.g., two-by-two matrix calculations in the SF approach) may shift students' focus from math to sense making and allow naïve students to allocate a larger mental effort on examining the features of the new paradigm when encountering the subject matter for the first time. In future phases of this research, we would like to study the possible justifications and variables that could affect students' learning more explicitly and in smaller grain sizes. We are also investigating whether utilizing SF approach in the sophomore-level course could lead to increased performance in upper division QM courses.
[1] H. Sadaghiani, Ph.D. thesis, The Ohio State Univer. (2005).

[2] J. Sweller, Cognitive science 12, (1988).

[3] Univ. of Washington \& Colorado QM Tutorials; C. Singh, Am. J. Phys. 76 (2008); C. Wieman et al. Phys. Teach. 48 (2010).

[4] G. Zhu and C. Singh, Am. J. Phys. 79, 499 (2011).

[5] Passante et al., Phys. Rev. Phys. Educ. Res. (2015).

[6] C. Manogue et al., PERC Proc., AIP (2012).

[7] H. Sadaghiani et al., PERC Proc., AIP (2015).

[8] H. Sadaghiani \& S. Pollock, Phys. Rev. Phys. Educ. Res. 11, 1 (2015).
[9] E. g., D. McIntyre, 1st ed. Pearson. (2011).

[10] E. g., D. Griffiths, 3rd ed., Prentice-Hall (2003).

[11] L. Bao, Ph. D. thesis, University of Maryland (1999).

[12] Thronton and Rox 4rd ed., Cengage Learning (2013).

[13] QMCA is available at: https://www.physport.org.

[14] A. Orton, Educ. Stud. Math. 14, 1-18 (1983).

[15] Mawer \& Sweller (1982) Sweller, Mawer, \& Howe (1982).

[16] S. McKagan et al., Phys. Rev. Phys. Educ. Res. 6, 1 (2010). 